\title{
RESISTANCE AGAINST MASS IMMIGRATION - An evolutionary explanation -
}

\author{
WERNER GUTH AND KLAUS RITZBERGER \\ University of Frankfurt, Germany, and \\ Institute for Advanced Studies, Vienna \\ January 1992
}

\begin{abstract}
In Europe we face very different living standards in different countries. In former times such discrepancies did not induce mass migration since mobility was restricted, especially between Eastern and Western European countries. But now the relatively richer countries are confronted with mass immigration and also strong resistance against it. We will show that resistance against mass immigration can be explained as being genetically determined. Specifically, we will analyse a very simple game model of immigration with an undetermined preference parameter deciding whether an incumbent engages into opposition against mass immigration or not. It is shown that preference for fighting against mass immigration is the only evolutionarly stable strategy for all possible parameter constellations. In our view this has important political implications regardless whether one wants to argue for a more liberal immigration low or against it.
\end{abstract}

\section{INTRODUCTION}

Drastic discrepancies in the living standard of different countries usually induce mass migration if international mobility is not restricted. Europe presently is facing enormous discrepancies in the living conditions of various countries, especially between Eastern and Western European countries. In former times the better living conditions in Western European countries did not induce mass immigration from East to West, since due to the Iron Curtain mobility between East and West Europe was practically non-existing. Fortunately this restriction of international mobility has disappeared. But, of course, one also has to expect mass migration from East to West Europe.

Although nearly all European countries and most of their citizens were very positive and enthusiastic about the dramatic political changes in the formerly communist countries, they often try to limit mass migration. Depending on the country and the political party the justifications for restricting mass immigration vary. In our view, there is, however, a basic and general reason, namely the strong resistance against mass immigration in most of the countries facing it. 
Now, resistance against invaders is a typical phenomenon in the animal kingdom where behavior is genetically determined. This fact implies that, at least in the animal kingdom, resistance against invaders seems to provide a greater reproductive success than a more liberal attitude to immigration. Here reproductive success is measured by the expected number of offsprings which decide about the future composition of the population. At least in the early stages in the development of mankind, which still determines our present genotype to a large extent, the situation must have been similar: Human genotypes, programmed to fight against invaders, earn a higher reproductive success than the more liberal ones. The main purpose of our paper is to provide a formal model and a methodology to analyse this conjecture more rigorously.

The model will be a simple game model which can be solved by a rather weak rationality requirement, namely repeated elimination of dominated strategies. Depending on the exogeneous parameters there are three generic solutions, called 'the liberal paradise', 'preventive resistance', and 'what we are afraid of.

The basic methodology is the one of evolutionary stability. Unlike in evolutionary biology, where genotypes directly determine behavior, we rely on a genetically encoded preference parameter which, together with living conditions, decides whether a given behavior is rational or not [see Guth and Yaari, 1990, as well as Guth, 1990, for similar studies]. Thus we can compute the solution for all possible constellation of preference parameters and derive the living conditions which this solution implies. Assuming that reproductive success depends on the living conditions we then can define an evolutionary game the strategies of which, or genotypes, are the possible preference parameters and the payoff function of which measures the reproductive success of the interacting genotypes.

To solve the evolutionary game we apply the concept of evolutionarily stable strategies [Maynard Smith and Price, 1973]. Although this concept is not always in line with dynamic stability of evolutionary processes, our result is unusually strong: The only evolutionarily stable strategy is strictly dominant and, therefore, the only optimal genotype irrespective of how the population is composed. This hold both under the requirement of an evolutionary stable strategy and under the evolutionary dynamics which we will also analyse.

According to our analysis 'the liberal paradise' can only be an episode due to short lived mutants or the absence of migration. Thus one has to expect either 'preventive resistance' or 'what we are afraid of. We discuss the parameters which influence this result and how they can be influenced politically. A genetically determined preference parame- 
ter does not exclude that phenotypical preferences can be influenced by education, personal experiences, and the political climate. To compensate the genetical predisposition one has, however, to exert a continuous opposing influence at least as long as the country is faced with mass migration, since every new generation might oppose to mass migration without this compensating influence.

The plan of the paper is as follows: Section II states the simple underlying game model to which in Section III the concept of an evolutionary stable strategy is applied. Section IV considers a fairly general class of dynamic evolutionary processes, reinforcing the results from Section III. Section IV draws conclusions.

\section{THE GAME MODEL}

We will model mass migration as an encounter of two individuals, one immigrant and one incumbent, who should be viewed as being members of large populations. The game model is very simple so that it can be solved by very basic, i.e. also non-controversial rationality requirements. Since the model is asymmetric, it will be symmetrised in Section III before we apply the concept of evolutionarily stable strategies.

Let player 1 be the potential immigrant of palyer 2's, the incumbent's, country or territory. The simple game is graphically illustrated in Figure II.1.

\section{Insert Figure II.1 about here}

A play starts at the origine $\mathcal{O}$ (the top decision node) with player 1's choice between $I$ and $S$ where $I$ stands for immigration and $S$ for staying. After $S$ the game ends immediately since players 1 and 2 do not meet. If, however, 1 enters 2 ' territory, player 2 can decide whether the engage into opposition against immigration (the move $F$ ) or not (the move $Y$ of player 2). In case of $Y$ the game ends with both players living on 2 's territory. If 2 has engaged into opposition against immigration, i.e. move $F$ by player 2 , it is decided by a chance move (of the chance player 0 ) whether 1 can become a final resident of 2's territory (the move $R$ with probability $w$ ) or not (the move $L$ with the positive complementary portability $1-w$ ).

The payoff vectors attached to the terminal nodes, i.e. the bottom nodes in Figure II.1, give the payoff of player 1 above and of player 2 below. Thus 1's choice of $S$ yields the living standard $p$ for player 1 and $r$ for player 2 , where we assume $r>p \geq 0$. The play $(I, Y)$ yields the same average living standard $(r+p) / 2$ for both players who are living in the same territory. The underlying assumption is, of course, that 2 
produces $r$ and 1 only $p$, regardless of whether he stays in his home country or migrates into 2's territory, and that individuals living in the same country have to share their resources equally.

In case of the play $(I, F, L)$ player 1 finally returns to his home country. Returning after an unsuccessful attempt to migrate may impose a cost $C \geq 0$ on player 1 . This $\operatorname{cost} C$ may or may not be related to player 1's living conditions. In fact, to determine evolutionart stable strategies, we will treat $C$ as deductions from the living standard of player 1, while in the dynamic analysis we will also allow for the possibility that $C$ is unrelated to living conditions. Thus after $(I, F, L)$ player 1's resulting living conditions are $p-C$ (or $p$ for the dynamic analysis), while player 2 will enjoy $r$. Since after the play $(I, F, R)$ player 1 becomes a final resident of 2's territory, he receives the same living standard $(r+p) / 2$ as after $(I, Y)$. For player 2 the living standard is also $(r+p) / 2$ of which we substract $P$. Again this cost $P$ may or may not bear directly on living conditions of player 2. For the analysis of evolutionart stable strategies we will assume that the preference parameter $P$ is purely unrelated to the living standard of player 2 (and, therefore, also to the reproductive success of player 2 ). For the evolutionary dynamics we will generalize this setup and allow for all possible impacts of $P$ on living conditions. The interpretation of a positive parameter $P$ is obviously that the incumbent hates to live together with somebody, whom he could not make leave. Similarly, a negative parameter $P$ would mean that 2 enjoys 1's company after his unsuccessful attempt to get him out. Here we do not impose any assumption for the parameter $P$, but try to derive its value in Section III by searching for the evolutionarily stable strategy $P$ [see Güth and Yaari, 1990, and Güth, 1990, who also rely on the idea of genetically determined preference parameters], i.e. the parameter $P$ is determined endogeneously, whereas the other parameters $p, r$, and $w \in(0,1)$ are exogeneously given. For the evolutionar $f$ stable strategies we will also treat $C$ as exogeneous, while for the evolutionary dynamics $C$ is endogeneous. Before starting our evolutionary analysis we want to derive the optimal behavior for both players for all generic parameter constellations $(p, r, C, w, P), r>p \geq 0, C \geq 0,1>w>0$.

Since the game of Figure II.1 is one with perfect information (the deciding player always knows all previous moves), the solution can be determined by repeated elimination of dominated strategies. Clearly, $F$ is better than $Y$ for player 2, if

$$
\frac{(1-w)(r-p)}{2 w}>P
$$

and $Y$ is better than $F$ if inequality (II.1) is reversed. In case of (II.1) 
player 1's move $I$ implies an expectation of

$$
w \frac{r+p}{2}+(1-w)(p-C)
$$

for player 1 which has to be compared with the living standard $p$ implied by 1 's choice of $S$. Thus $I$ is better than $S$ for player 1 if

$$
\frac{w(r-p)}{2(1-w)}>C
$$

whereas the opposite is true for the reversed inequality.

If inequality (II.1) is reversed, the analogous condition that $I$ is better than $S$ is $r>p$ and therefore always satisfied.

The non-generic parameter constellations $(p, r, C, w, P)$ are those with equality in (II.1) or (II.3). These highly specific constellations are not relevant for our evolutionary analysis and can therefore be neglected.

\section{Insert Figure II.2 about here}

In Figure II.2 we give a graphical illustration of the three possible generic solutions $(I, F),(S, F)$, and $(I, Y)$ in the $(P, C)$-plane. Wherever the opposite of inequality (II.1) is true, we are in the region $(I, Y)$, called 'The liberal paradise' since 1 immigrates and 2 does not resist against l's immigration.

For inequality (II.1) the result depends on whether (II.3) is satisfied or not. If (II.1) and (II.3) are true, we are in the region $(I, F)$ where 1 migrates although he knows 2 will try to keep him out. Because of this unfortunate struggle between the incumbent and the invader we refer to region $(I, F)$ as to 'What we are afraid of'.

The final region $(S, F)$ results from (II.1) and the reverse of inequality (II.3). Here 2 is determined to fight against immigration and induces thereby player 1 to abstain from immigration, i.e. player 2's willingness to fight against immigration is a preventive threat. This explains why the solution region $(S, F)$ is named 'Preventive resistance'.

The following evolutionary analysis will anticipate these results for all generic parameter constellations $(p, r, C, w, P)$ and try to determine the evolutionarily stable strategy $P$.

\section{Evolutionary Stable Strategy}

To apply the concept of evolutionarily stable strategies [Maynard Smith and Price, 1973, see also Selten, 1983, and van Damme, 1987] the encounter of an invader and an incumbent has to be modelled 


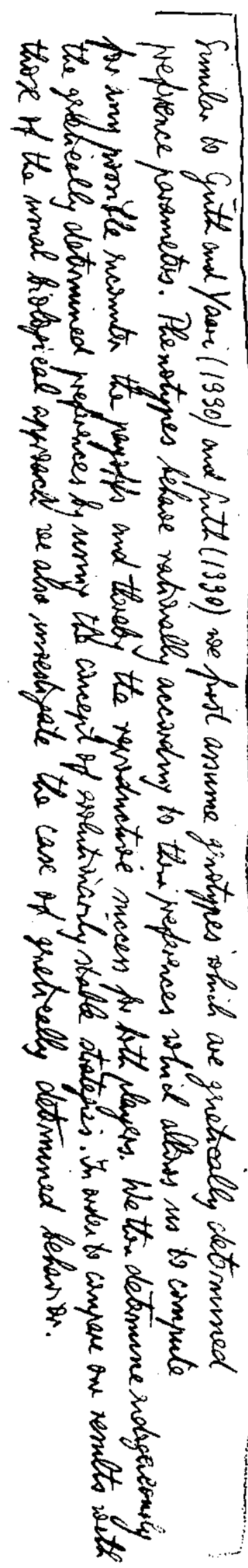

symmetrically. In evolutionary biology a genotype has to specify a behavior both for the invader and the incumbent since a phenotype can be in both positions. Furthermore, symmetry can be easily established by introducing an initial chance move which determines with equal probability whether player 1 or player 2 is living in the relatively richer country and, therefore, threatened by immigration of the other player. The game in Figure III. 1 is symmetric, also in the more restrictive sense of evolutionary game theory [Selten, 1983], since every move in the subgame after the left top chance move with probability $1 / 2$ can be identified with a move in the subgame after the right top chance move with probability 1/2. In Figure III.1 this identification is indicated by assigning the same symbol $I, S, F, Y, R$, or $L$ to both moves.

Insert Figure III.1 about here

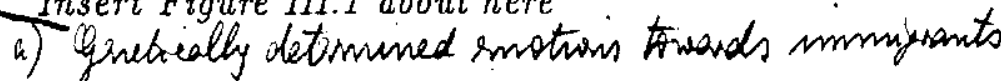

Assume an arbitrary constellation $(p, r, C, w)$ of the exogeneously determined parameters. We want to derive for $(p, r, C, w)$ and all values of $P$ the living conditions for both players which are assumed to determine their reproductive success. The underlying idea is that at least in the early stages in the development of mankind the available resources determined decisively the expected number of offspring $\$$ and thereby the future composition of the population.

According to Figure II.2 the solution of the game and thereby the living conditions of both players depend on $P$ only in the sense that for $P$ greater than

$$
P^{*}=\frac{(1-w)(r-p)}{2 w}
$$

the solution is $(I, Y)$, i.e. 'the liberal paradise', whereas for $P<P^{*}$ it is either $(S, F)$ or $(I, F)$. Of course, $P$ may very well depend on phenotypical characteristics which can account for different values of $P$ in the range $P>P^{*}$ or $P<P^{*}$. Here we will simply assume that the endogeneous parameter $P$ can take only two values, namely $P^{+}$ with $P^{+}>P^{*}$ and $P^{-}$with $P^{-}<P^{*}$. By this assumption we also exclude the degenerate case $P=P^{*}$ which is of no relevance at all for the evolutionary analysis.

The evolutionary analysis is based on the symmetric normal form game $G=\left(\left\{P^{+}, P^{-}\right\} ; H\right)$, where $\left\{P^{+}, P^{-}\right\}$is the set of strategies or genotypes; for the case at hand it is the set of possible values of the endogeneous preference parameter $P$. Both players 1 and 2 have the same strategy set since they are both members of the same species. $H$ is the payoff function which determines the reproductive success of 
both players for all possible strategy combinations $\left(P_{1}, P_{2}\right)$ with $P_{i} \in$ $\left\{P^{+}, P^{-}\right\}$for $i=1,2$. Since the game is symmetric, it is sufficient to specify the reproductive success of player 1 which will be denoted by $H\left(P_{1}, P_{2}\right)$. For player 2 the reproductive success from the strategy combination $\left(P_{1}, P_{2}\right)$ is given by $H\left(P_{2}, P_{1}\right)$.

A strategy $P \in\left\{P^{+}, P^{-}\right\}$is called evolutionarily stable [Maynard Smith and Price, 1973], if it satisfies the following two conditions:

$$
\begin{aligned}
& \text { (i) } H(P, P) \geq H\left(P_{1}, P\right), \quad \forall P_{1} \in\left\{P^{+}, P^{-}\right\}, \\
& \text {(ii) } \left.\left.\sharp P_{1} \in\left\{P^{+}, P^{-}\right\} \backslash\{P\}\right\}^{\mp} H(P, P)=H\left(P_{1}, P\right)\right] \\
& \Longrightarrow H\left(P, P_{1}\right)>H\left(P_{1}, P_{1}\right) .
\end{aligned}
$$

Condition (i) requires that the genotype $P$ must be optimal in a monomorphic population consisting only of $P$-genotypes. In other words: In a $P$-monomorphic population no other genotype can have more offspring $\$$ than $P$. Observe that condition (i) does not rule out the case $H(P, P)=H\left(P_{1}, P\right)$ for some alternative strategy $P_{1}$. In such a case $P$ must be a better reply against $P_{1}$ than $P_{1}$ itself, i.e. in a population containing only $P_{1}$-genotypes or $P_{1}$ - and $P_{1}$-genotypes the strategy $P$ has a greater reproductive success than $P_{1}$.

The static concept of evolutionarily stable strategies may not always coincide with dynamic stability of evolutionary processes Weissing, 1991, for a discussion]. For the simple case at hand this, however, cannot occur since the only evolutionarily stable strategy $P$ is strictly dominant, i.e. $P$ earns a higher reproductive success than the other strategy for all values of $P_{2}$. In this section, where we focus on $P$ the return-fare of an invader, $C$, will be deducted from living conditions, while $P$ will be taken to be a pure preference parameter, entirely unrelated to living conditions.

To determine the payoff function $H$ of $G$ for the case at hand we distinguish two cases, namely whether (II.3) is satisfied or whether the converse condition

$$
\frac{w(r-p)}{2(1-w)}<C
$$

is true.

a. The case (II.3). If the incumbent's strategy is $P^{+}$, the solution is $(I, Y)$ according to Figure II.2, i.e. both players receive the reproductive success $(r+p) / 2$. For the incumbent's strategy $P^{-}$, the solution is $(I, F)$ which yields the reproductive success

$$
(1-w)(p-C)+w \frac{r+p}{2}
$$


for the invader and

$$
(1-w) r+w \frac{r+p}{2}
$$

for the incumbent, since we are in case (II.3) and since the payoff parameter $P$ is assumed unrelated to the living conditions and the reproductive success of the incumbent. Our results are summarized by Table III.1 which determines the reproductive success $H\left(P_{1}, P_{2}\right)$ of player 1 for all four possible strategy combinations $\left(P_{1}, P_{2}\right)$. Since both players have equal chances for becoming the incumbent, $H\left(P_{1}, P_{2}\right)$ is determined with probability $1 / 2$ both by $P_{1}$ and $P_{2}$.

\section{Insert Table III.1 about here}

Due to $1>w$ and $r>p$ the following two inequalities are true:

$$
\frac{r+p}{2}<(1+w) \frac{r+p}{4}+(1-w) \frac{r}{2}
$$

and

$$
(1+w) \frac{r+p}{4}+(1-w) \frac{p-C}{2}<(1-w) \frac{r+p-C}{2}+w \frac{r+p}{2} .
$$

Conditions (III.5) and (III.6) imply that $P^{-}$is strictly dominant. Since there can be no alternative best reply in addition to $P^{-}$, this proves:

LEMMA III.I. If the constellation $(p, r, C, w)$ of exogeneous parameter satisfies condition (II.3), then the strategy $P^{-}$is strictly dominant and therefore the only evolutionarily stable strategy of game $G$.

b. The case (III.2). The only difference results for the incumbent's strategy $P^{-}$which implies the solution $(S, F)$ according to Figure II.2. The reproductive sucess implied by $(S, F)$ is $r$ for the incumbent and $p$ for the potential invader. The reproductive sucess for all four possible strategy constellations $\left(P_{1}, P_{2}\right)$ in game $G$ is illustrated in Table III.2, similarly to Table III.1 for the previous case.

\section{Insert Table III.2 about here}

Clearly, due to $r>p$ the strategy $P^{-}$is strictly dominant which proves:

LEMMA III.2. If the constellation $(p, r, C, w)$ of exogenous parameters satisfies condition (III.2), then the strategy $P^{-}$is dominant and, therefore, the only evolutionarf stable strategy of the game $G$.

Combining the two Lemmas yields: 
THEOREM 1. In the evolutionary game $G=\left(\left\{P^{+}, P^{-}\right\}, H\right)$, the payoff function $H$ Which is given in Table III.1 for (II.3), respectively in Table III.2 for (III.2), the strategy $P^{-}$is the only evolutionard stable strategy for all constellations $(r, p, C, w)$ of exogenous parameters satisfying $r>$ $p$ and $1>w$.

The assumption $r>p$ is no restriction at all, since this is the only reason for mass migration within the limited scope of our model. Thus according to the Theore above we only rule out the highly specific borderline case $w=1$ meaning that the incumbent cannot make the invader leave at all. Since for $w=1$ immigration will always imply the same reproductive success $(r+p) / 2$ for both players, there can be no evolutionary drive for the endogenous paramter $P$ in the limiting case $w=1$. Or in other words: For $w=1$ resistance against mass immigration does not make sense at all. Thus the two conditions, $r>p$ and $1>w$, are implied by our research problem and no real restrictions to our results. In this sense the Theorep above can be rephrased by saying that $P^{-}$is the only evolutionarily stable strategy regardless of how the exogenous game parameters $p, r, C$, and $w$ are specified.

Our results assume, of course, that all strategically relevant aspects of the conflict between the incumbent and the invader are captured by our basic game model in Figure II.1.. We do not wish to claim that this is an entirely realistic assumption. However, a similar anlysis can be performed for other game models of conflicts between an incumbent and an invader.

Even, if one believes that Figure II.1. is an adequate representation of the conflict, we readily admit that Theorem 1 is only a special result. First of all only the preference parameter $P$ is to evolution. Clearly, the parameter $C$ which measures, how an unsuccessful invader suffers, could also be considered as being subject to genetical evolution. For the evolutionary game of Figure III. 1 one could rely on the mutant space $(P, C)$ which contains all possible values of the incumbent's preference parameter $P$ and the invader's cost parameter C.

Furthermore, the assumption that $P$ is entirely unrelated to reproductive success is by no means necessary for our approach. One could easily generalise the analysis by allowing that a certain proportion of $P$ is related to reproductive success, whereas the remaining part is not. Similarily, the more general case of mutants $(P, C)$, described above, can be analysed by requiring that only a certain proportion of $P$ and a possibly different proportion of $C$ are unrelated to reproductive success, where both proportions can range from 0 to 1 . 
Another restriction of Theorem 1 is that both interacting individuals are certain about the incentive structure of their respective opponent. In agenetically mixed population this implicitly assumes that the opponent's type can be recognized, once the opponent is faced. This need not always be true, since a certain P-type might be able to imitate another P-type, if this is profitable. This shows that the above results implicitly rely on perfect signalling of the respective types.

Again, we readily admit that perfect signalling may be highly unrealistic. However, the assumption of perfect signalling is by no means necessary for our fundamental approach. It could easily be generalised to situations, where none of the two encountering individuals is certain about the opponent's type. If signalling is impossible, it seems natural to assume that both individuals know by experience only the distribution of the population, but not the specific type of their actual encounter. In such a scenario the beliefs concerning the other's type are determined by the frequencies of types in the present population.

Here we do not attempt to generalise our analysis along these lines, since we expect similar results. What we will do instead is to generate some support for the result from the static concept of evolutionary stable strategies by explicitely analysing the dynamics of evolutionary processes. Such processes can describe the dynamics of genetical and also of cultural evolution of human populations. There are, however, at least two important differences between genetical and cultural evolution. First, genetical evolution is much slower. Second, cultural evolution does not necessarily require symmetry, as imposed in Figure III.1.. We do not wish to exclude the possibility that our dynamic analysis is interpreted as a model of cultural evolution. Therefore, in the sequel we go back to the original game model of Figure II.1., instead of analysing the symmetric encounter in Figure III.1..

\section{EVOLUTIONARY DYNAMICS}

Let us now turn to an alternative view on the problem of the evolution of preferences. In Section III we had to symmetrize the game from Section II in order to be able to apply the concept of an evolutionary stable strategy. Although this is common in biology, it may not be entirely convincing, when applied to the problems currently facing

Europe. Thus in the present section we offer an asymmetric alternative whichjjn biological terms could be viewed as an evolutionary analysis of the interaction of two different species. Leaving the game from Section II asymmetric as it is, however, forces us to be more explicit on the evolutionary dynamics (as opposed to the static concept of evolutionary stable strategies). 
Still we will stick to the description of preferences by the two parameters $C$ and $P$. But now both preference parameters are endogeneous: $C$ describes the costs, or rather: perception of costs, of "loosing a battle" to player 1, while $P$ describes player 2's perception of the costs associated with loosing a conflict. In particular, imagine that there are two populations, one of potential migrants and one population of incumbents. Both populations are very large and the individuals, of which a given population consists, are identical, except for their preference parameters concerning their perception of the costs of loosing a fight. These preference parameters may, however, not even influence the living standards of individuals and, therefore, their reproductive success. The extent to which preferences, measured by $C$ for potential migrants and by $P$ for incumbents, impact on living conditions is measured by a parameter $\delta$, $0 \leq \delta \leq 1$. If $\delta=0$, then preferences have no impact on the reproductive success of individuals at all, if $\delta=1$, then $C$ and $P$ are fully deducted from the living standards. Since we make no further assumption on $\delta$, both cases are covered by our analysis. As the interested reader can readily verify, we could even assume different $\delta$ 's for incumbents and potential immigrants. Since nothing would be changed in our analysis by such an asymmetry of the $\delta$ 's, we have sacrificed this generalisation for brevity.

Since individuals in a given population are ipdentical except for preferences and their reproductive success depends on living conditions which may be unrelated to preferences, the evolution of the two populations is determined by the success, measured in terms of living standards, of certain preference types in the interaction of the two populations. Given our assumptions, the two populations can be fully described by the joint distribution $\Phi(C, P)$ of preference parameters, where $C$ is the parameter relevant for potential migrants and $P$ is the parameter relevant for incumbents. Let $\phi(C, P)$ be the density corresponding to the distribution $\Phi(C, P)$ and define the marginal densities 1

$$
f_{t}(C)=\int_{-\infty}^{\infty} \phi_{t}(C, P) d P, \quad g_{t}(P)=\int_{-\infty}^{\infty} \phi_{t}(C, P) d C .
$$

We assume that each period pairs of individuals, each pair consisting of one potential migrant and one incumbent, are chosen at random to play essentially the game from Figure II.1.. The random matching technology is such that the probability $\pi_{t}$ that a pair, consisting of a potential migrant with cost parameter $C$ and an incumbent with cost parameter $P$, is matched is given by

$$
\pi_{t}(C, P)=f_{t}(C) g_{t}(P) .
$$


Thus the matching technology is fair in the sense that it only depends on the marginal densities, such that the conditional probability that a potential migrant (incumbent) with parameter $C(P)$ will meet on incumbent (migrant) with cost parameter $P(C)$ does not depend on $C$ $(P)$, i.e. $\pi_{t}(P \mid C)=g_{t}(P)$ and $\pi_{t}(C \mid P)=f_{t}(C)$. Define

$$
\begin{aligned}
& G_{t}(y)=\int_{-\infty}^{y} g_{t}(P) d P, \\
& F_{t}(x)=\int_{-\infty}^{x} \underset{t}{f}(C) d C .
\end{aligned}
$$

In contrast to Section II we, however, assume here that the two players matched cannot verify each otheff type, that is: The potential migrant knows his parameter $C$, but not the preference parameter of the incumbent with whom he is matched, and the incumbent knows his $P$, but not the preference parameter of the migrant he confronts. Thus each pair plays the game from Section II under incomplete information about each othef cost parameter.

Still the incumbent's problem does not differ very much from player 2 's problem in Section II. If the incumbent's $P$ satisfies

$$
P>P^{*}=\frac{(1-w)(r-p)}{2 w}
$$

then he will play $Y$ and obtain a living standards $(r+p) / 2$, if the potential migrant immigrates, and living standard $r$, if the potential migrant plays $S$. If the inequality is reversed, $P<P^{*}$, then the incumbent is determined to play $F$, if the migrant plays $I$. The potential migrant's problem is slightly more complicated, because he has to take into account both his $C$ and the probability to be in a match with an incumbent, who satisfies $P>P^{*}$. From (IV.3) the probability to meet an incumbent with $P>P^{*}$ is given by $1-G\left(P^{*}\right)$, such that a potential migrant will decide to stay home, if his $C$ satisfies

$$
C>\frac{\left[1-(1-w) G_{t}\left(P^{*}\right)\right](r-p)}{2(1-w) G_{t}\left(P^{*}\right)}
$$

Define the function $x:[0,1] \rightarrow \Re_{+} \cup\{\infty\}$ by

$$
x(a)=\frac{[1-(1-w) a](r-p)}{2(1-w) a}, \quad \forall a \in(0,1]
$$


and by $x(0)=+\infty$. With the help of this function, the expected living conditions of a potential migrant with preference parameter $C$, denoted for given $F$ and $G$ by $u_{1}(C)$, can be written as

$$
u_{1}^{t}(C)=\left\{\begin{array}{l}
p \text { if } C>x\left(G_{t}\left(P^{*}\right)\right) \text { and } G_{t}\left(P^{*}\right)>0 \\
G\left(P^{*}\right)\left[p+w \frac{r-p}{2}-\delta(1-w) C\right]+\left[1-G_{t}\left(P^{*}\right)\right] \frac{r+p}{2} \\
\quad \text { otherwise }
\end{array}\right.
$$

Recall that $\delta$ is the parameter which measures how strongly the preference parameters influence living standards. The expected living conditions of an incumbent with preference parameter $P$ can be written as

$$
\stackrel{t}{u_{2}(P)}=\left\{\begin{array}{l}
r-w F_{t}\left(f\left(G\left(P^{*}\right)\right]\right)\left[\frac{r-p}{2}+\delta P\right], \text { if } P \leq P^{*}, \\
r-F_{t}\left(f\left(G\left(P^{*}\right)\right]\right) \frac{r-p}{2}, \text { if } P>P^{*} .
\end{array}\right.
$$

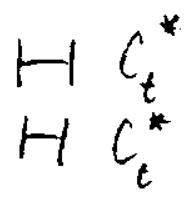

Observe that both $u_{1}^{t}(C)$ and $u_{2}^{t}(P)$ are continuous. The average living conditions in the two populations, given $F_{l}$ and $G_{t}$, are denoted by $U_{1}^{t}$ and $U_{2}^{\leftarrow}$,

$$
U_{1}^{t}=\int_{-\infty}^{\infty} u_{1}^{t}(C) d F(C), \quad U_{2}^{t}=\int_{-\infty}^{\infty} u_{2}^{t}(P) d G_{t}(P) .
$$

These functions can be written as

$$
U_{1}^{t}=\left\{\begin{array}{c}
p+(1-w) G\left(P^{*}\right)\left[x\left(G\left(-P^{*}\right)\right] F\left(t\left(G\left(P^{*}\right)\right)\right)-\right. \\
-\delta\left[\int_{-\infty} C d F(C)\right], \text { if } \underset{t}{G\left(P^{*}\right)>0,} \\
\frac{r+p}{2}, \text { if } G_{t}\left(P^{*}\right)=0
\end{array}\right.
$$

$$
\begin{gathered}
\frac{t-1}{2}\left(1-G\left(P^{*}\right)(1-w)\right) \\
H c_{t}^{*}
\end{gathered}
$$

and as

$$
U_{2}^{t}=\left\{\begin{array}{c}
r-F_{t}\left(x\left(\not\left(P^{*}\right)\right)\right]\left[\left(1-(1-w) G\left(P^{*}\right)\right) \frac{r-p}{2}-\right. \\
\left.-\delta w \int_{-\infty}^{P^{*}} P d G(P)\right], \text { if } G\left(P^{*}\right)>0 \\
\frac{r+p}{2}, \text { if } G\left(P^{*}\right)=0
\end{array}\right.
$$

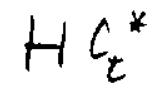

with $U_{1}^{t}$ and $U_{2}^{t}$ again continuous. Thus both individual as well as average living conditions depend on the two marginal distributions of the preference parameters in the two populations. We will now attempt f $\phi$ study the evolution of $F_{t}$ and $G_{t}$ over time.

Let $\mathcal{H}$ be the set of all continuously differentiable distributions on $\Re^{2}$. Then $\Phi_{t} \in \mathcal{H}$ and an evolutionary process can be defined as a mapping $\varphi: \mathcal{H} \times \Re_{+} \rightarrow \mathcal{H}$ or $\left(\Phi_{o}, t\right) \mapsto \Phi_{t}$. Suppose the distribution of the 
preference parameters in the two populations at some point in time $t$ is given by $\Phi(C, P)$ with marginal densities $f_{t}(C)$ and $g_{t}(C)$. Then the family of evolutionary processes which we will consider is the family of processes which satisfy

$$
\begin{aligned}
& \dot{f}_{t}(C)<0 \Longleftrightarrow f_{t}(C)\left[u_{1}^{t}(C)-U_{1}^{t}\right]<0, \\
& \dot{g}_{t}(P)>0 \Longleftrightarrow g_{t}(P)\left[u_{2}^{t}(P)-U_{2}^{t}\right]>0 .
\end{aligned}
$$

This is the family of evolutionary processes such that, if some value of the preference parameter which is still present in the population generates worse living conditions than what obtains on average in the respective population, then the (marginal) frequency of this value of the preference parameter shrinks over time. On the other hand, if some value of the preference parameter results in superior living conditions as compared to the population average, then the (marginal) frequency of this value of the parameter will increase over time, provided it is still present in the population. A quasi-stationary distribution $\Phi \in \mathcal{H}$ is one which satisfies $\dot{f}(C)=\dot{g}(P)=0$, for all $(C, P)$ in the support of $\Phi$.

THEOREM 2. (i) Whenever $0<G_{0}\left(P^{*}\right)<1$ and $0<F_{0}\left(G_{0}\left(P^{*}\right)\right)<1$ holds, then for any $\varepsilon>0$ there is some $t_{\varepsilon}>0$ such that $G_{t}\left(P^{*}\right) \geq 1-\varepsilon$ and $F_{t}(x(1-\varepsilon)) \geq 1-\varepsilon$ for all $t \geq t_{\varepsilon}$.

(ii) Any quasi-stationary distribution satisfies either

$$
G\left(P^{*}\right)\left[>0=F\left(x\left(G\left(P^{*}\right)\right)\right) \text { or } G\left(P^{*}\right)=1=F(x(1))\right. \text {. }
$$
Proof: (i) Assume that for some $t \geq 0$ one has $0<G_{t}\left(P^{*}\right)<1$ and
$0<F_{t}\left(G_{t}\left(P^{*}\right)\right)<1$. From $F_{t}\left(G_{t}\left(P^{*}\right) H\right)<1$ it follows that there $C_{t}^{*}$ exists some $C>(G)\left(P^{*}\right)$ for which $f_{t}(C)>0$. Using (IV.4) and (IV.6.1) one has for this $C$ that

$$
\begin{gathered}
u_{1}(C)-U_{1}=(1-w) G_{t}\left(P^{*}\right)\left[\delta \int_{-\infty}^{\left.t\left(P^{*}\right)\right)} C d F_{t}(C)-\right. \\
\left.-p\left(G_{t}\left(P^{*}\right)\right) F_{t}\left(x\left(G_{t}\left(P^{*}\right)\right)\right]\right]<0,
\end{gathered}
$$

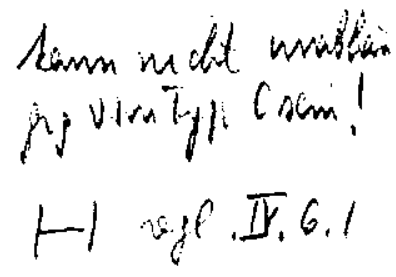

where the final strict inequality follows from $\Phi_{t} \in \mathcal{H}$ which implies that $F_{t}$ cannot have any atoms, such that $F_{t}\left(G_{t}\left(P^{*}\right)\right)>0$ implies that there is some $C<b\left(G_{t}\left(P^{*}\right)\right.$ for which $f_{t}(C)>0$. We conclude from (IV.7) that $\dot{f}_{t}(C)<0$ for all $C>\oint\left(G_{t}\left(P^{*}\right)\right.$ which are still present in the population, $f_{t}(C)>0$.

On the other hand $G_{t}\left(P^{*}\right)<1$ implies that these exists $P>P^{*}$ such that $g_{t}(P)>0$, and for any such $P$ one obtains from (IV.5) and (IV.6.2) 
that

$$
\begin{aligned}
& u_{2}(P)-U_{2}=\delta w F_{t}\left(x\left(G_{t}\left(P^{*}\right)\right)\right) \int_{-\infty}^{P^{*}} P d G_{t}(P)- \\
&-(1-w) G_{t}\left(P^{*}\right) F_{t}\left(x\left(G_{t}\left(P^{*}\right)\right)\right) \frac{r-p}{2}= \\
&=w F_{t}\left(x\left(G_{t}\left(P^{*}\right)\right)\right)\left[\delta \int_{-\infty}^{P^{*}} P d G_{t}(P)-P^{*} G_{t}\left(P^{*}\right)\right]<0,
\end{aligned}
$$

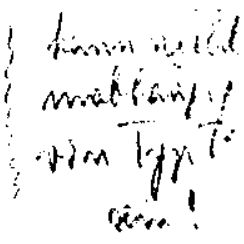

where the final strict inequality again follows from $\Phi_{t} \in \mathcal{H}$, because then $G_{t}$ cannot have any atoms such that $G_{t}\left(P^{*}\right)>0$ implies that there is some $P<P^{*}$ such that $g_{t}(P)>0$. Thus from (IV.7) one obtains $\dot{g}_{t}(P)<0$ for all $P>P^{*}$ for which $g_{t}(P)>0$.

Under the assumption $0<G_{0}\left(P^{*}\right)<1$ and $0<F_{0}\left(x\left(G_{0}\left(P^{*}\right)\right)\right)<1$ and for given $\varepsilon>0$ let $t_{1}$ denote the smallest $t \geq 0$ such that $G_{t}\left(P^{*}\right) \geq$ $1-\varepsilon$. By the above argument $t_{1}$ ist finite. Also by the above argument there is some finite $t_{2} \geq 0$ such that $F_{t_{2}}\left(x\left(G_{t_{2}}\left(P^{*}\right)\right)\right) \geq 1-\varepsilon$. Since $x$ is decreasing, the latter inequality implies $F_{t}\left(x\left(G_{t}\left(P^{*}\right)\right)\right) \geq 1-\varepsilon$ for all $t \geq \max \left(t_{1}, t_{2}\right)$. Setting $t_{\varepsilon}=\max \left(t_{1}, t_{2}\right)$ thus proves our claim.

(ii) Under quasi-stationarity $F\left(x\left(G\left(P^{*}\right)\right)\right)<1$ implies $G\left(P^{*}\right)>0$, because otherwise $G\left(P^{*}\right)=0$ would imply $x\left(G\left(P^{*}\right)\right)=\infty$ which would imply $F\left(x\left(G\left(P^{*}\right)\right)\right)=1$. Also $F\left(x\left(G\left(P^{*}\right)\right)\right)<1$ implies from $\Phi \in \mathcal{H}$ that there exists some $C>x\left(G\left(P^{*}\right)\right)$ such that $f(C)>0$. For this $C$ one would, under quasi-station $\$$ rity, have to the

$$
\begin{gathered}
u_{1}(C)-U_{1}=(1-w) G\left(P^{*}\right)\left[\delta \int_{-\infty}^{x\left(G\left(P^{*}\right)\right)} C d F(C)-\right. \\
\left.-x\left(G\left(P^{*}\right)\right) F\left(x\left(G\left(P^{*}\right)\right)\right)\right]=0
\end{gathered}
$$

But the latter can only hold, if $F\left(x\left(G\left(P^{*}\right)\right)\right)=0$. Thus the first possibility of a quasi-stationary distribution is given by $G\left(P^{*}\right)>0=$ $F\left(x\left(G\left(P^{*}\right)\right)\right)$.

If, on the other hand, $F\left(x\left(G\left(P^{*}\right)\right)\right)=1$, but $G\left(P^{*}\right)<1$, then there exists some $P>P^{*}$ for which $g(P)>0$ such that

$$
\delta \int_{-\infty}^{P^{*}} P d G(P)-P^{*} G\left(P^{*}\right)=0
$$

would have to hold under quasi-stationarity. From $\Phi \in \mathcal{H}$ it follows, however, that the latter inequality cannot be satisfied. Thus $F\left(x\left(G\left(P^{*}\right)\right)\right)=$ 1 implies under quasi-stationarity that $G\left(P^{*}\right)=1$. Thus the second 
possibility of a quasi-stationary distribution is given by $G\left(P^{*}\right)=1=$ $F(x(1))$.

What the Theorem says can be rephrased as follows: If the initial distribution of preferences is such that some migration at least will take place, $F_{0}\left(x\left(G_{0}\left(P^{*}\right)\right)\right)>0$, then within finite time any evolutionary process from the family (IV.7) will lead to a distribution of preferences which has almost all its mass concentrated on the region "that we are afraid of". Moreover, any distribution $\Phi$, whose marginal densities remain unchanged by evolutionary forces ("quasi-stationarity"), must either be concentrated entirely on the region "that we are afraid of", or must satisfy that virtually no migration at all takes place, $F\left(x\left(G\left(P^{*}\right)\right)\right)=0$.

\section{INTERPRETATION AND POLITICAL CONCLUSIONS}

Although $P^{-}<P^{*}$ is the only evolutionarily stable strategy of Section III for all reasonable constellations $(r, p, C, w)$ of exogeneous parameters and only $P<P^{*}$ can be dynamically stable in Section IV the stable genotype is not completely independent of the exogeneous parameters since $P^{-}<P^{*}$ and since $P^{*}$ is determined by these paramefts according to equation (III.1).

Now the parameter $P$ describes the feelings of the incumbent after an unsuccessful attempt to throw out the invader. Whereas a positive value of $P$ expresses anger, a negative value of $P$ can be interpreted as enjoying a multi-cultural society. Our analysis has shown that it is not the sign of $P$ which matters. Thus a political debate whether a multicultural society is good or bad might miss the point. What matters is whether $P$ is larger or smaller than $P^{*}$, since, as illustrated Figure II.2, behaviour is crucially different for the case $P>P^{*}$ for which the 'liberal paradise' results and for $P<P^{*}$, where the incumbent always threatens to throw out the invader.

The positive critical parameter value $P^{*}$ depends positively on the discrepancy $r-p$ of the prior living standard as well as on the relative probability $(1-w) / w$ by which an invader will leave, when he faces resistance against immigration. Many political measures currently taken in Western European countries are attempts to reduce the discrepancy in living conditions. The mob, responsible for riots against immigrants in Germany in 1991, is definitely trying to increase the relative probability $(1-w) / w$. But also more restrictive requirements for being accepted as an immigrant which are frequently proposed, can be viewed as measures aiming at an increase of $(1-w) / w$.

But, of course, such a discussion assumes that the parameter $P$ is given whereas $P^{*}$ can still be influenced politically. Our evolutionary analysis shows that the 'liberal paradise', i.e. $P>P^{*}$, can only be an 


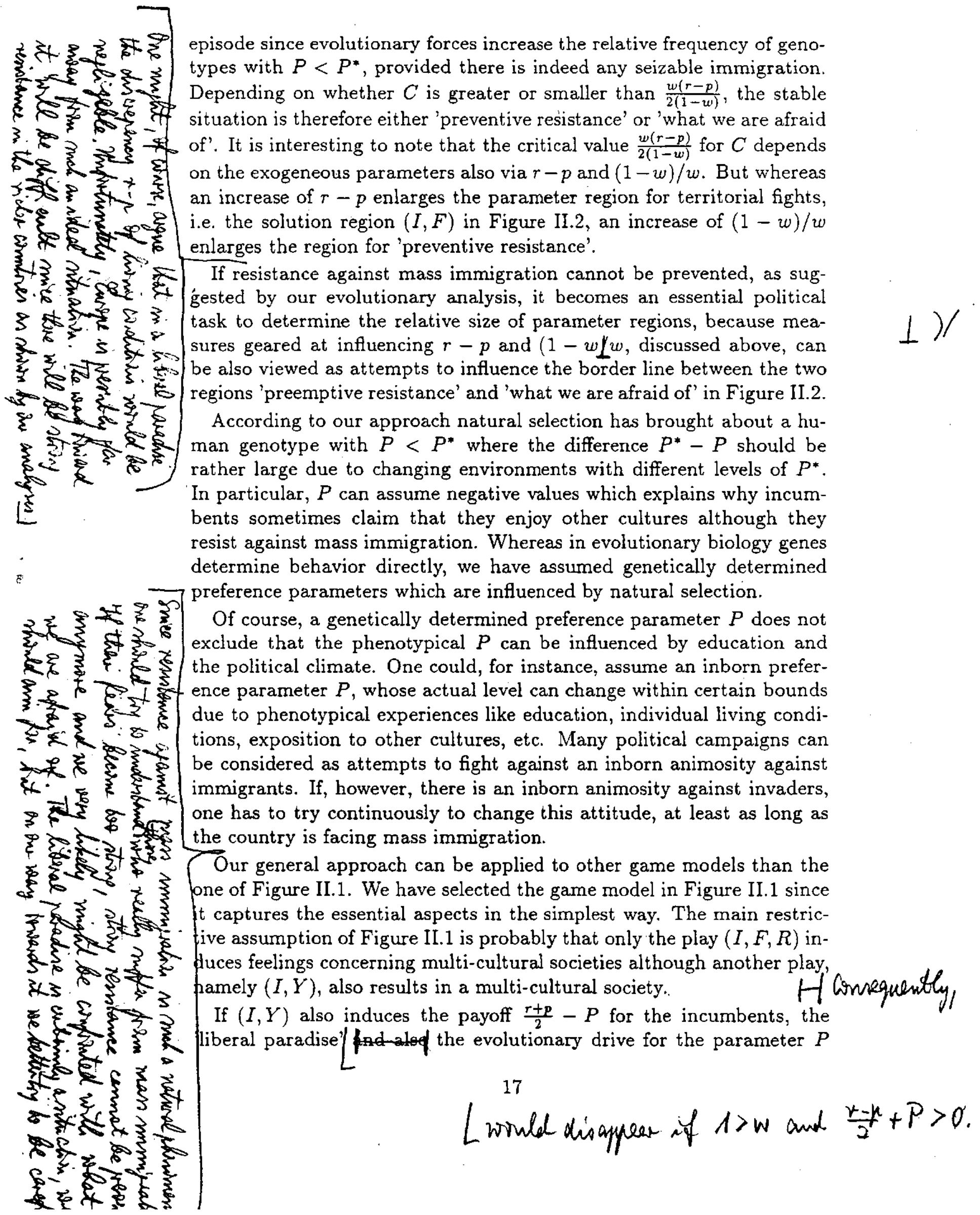




\section{$H$ trovids $P^{-}$wruld become sesn stringer}

weuld-depend on the probability-w that-an-immigrant-wins the-fight. Thus Figure II.1 seems to capture the more interesting situation where emotions concerning immigrants are triggered by unsuccessful resistance against mass immigration.

In our view, such an assumption can be defended. Many developed countries have experienced massive immigration movements without serious political debates, especially in situations of high employment. This indicates that emotions concerning immigration become virulent only if one has been strictly opposed to it but unable to prevent it.

If animosity against immigrants or sympathy for a multi-culture society is triggered by unsuccessfull resistance against mass immigration, behavior will depend crucially on the political climate which is not only influenced by the political parties but also by the mass media. A political debate, like the one in Germany in 1991, might trigger emotions which will seriously endanger the prospects for a peaceful life in a multicultural society.

Campaigns aiming at measures against mass immigration may help to win the next election, but may also induce long lasting animosity against immigrants, possibly also against earlier immigrants, who up to now did not encounter any resistance.

If mankind has an inborn tendency to resist against mass immigration, this can be politically exploited, especially by extremely rightist parties. To avoid this, one may want to limit immigration. Actually this is what we observe in all developed countries which partly rely on 'preventive resistance 'and partly experience 'what we are afraid of'. As predicted - by ouf study, 'the liberal paradise' does not/survive in the the simplistic framework of our moded

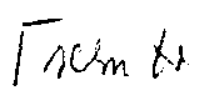

Of course, one may argue that in case of the 'liberal paradise' the discrepancy $r-p$ in living conditions would vanish, i.e. 'the liberal paradise' could be responsible for equal living conditions in countries without entry barriers. And this is perhaps the major lesson concerning the responsibility of economists: To convince the public that in-equalization of living standards is the most effective way to avoid political unrest.

\section{REFERENCES}

Güth W., Incomplete Information about Reciprocal Incentives - An Evolutionary Approach to Explaining Cooperative Behavior, unpubl. manuscript (January 1990).

Güth W. and M. Yaari, Explaining Reciprocal Behavior in Simple Strategic Games: An Evolutionary Approach, unpubl. manuscript (1990).

Maynard Smith J. and G.R. Price, The Logic of Animal Conflict, Nature 246 (1973), 15-18. 
Selten R., Evolutionary Stability in Extensive 2-Person Games, Mathematical Social Sciences 5 (1983), 269-363.

van Damme E., "Stability and Perfection of Nash Equilibria," Springer Verlag, 1987.

Weissing F.J., Evolutionary Stability and Dynamic Stability in a Class of Evolutionary Normal Form Games, in "Game Equilibrium Models I: Evolution and Game Dynamics," ed. by R. Selten, Springer Verlag, 1991.

Keywords. Migration, Evolution, Games

Werner Guth, Johann Wolfgang Goethe-Universitat, FB Wirtschaftswissenschaften, Mertonstr. 17, Postfach 111932, D-6000 Frankfurt am Main, Germany

Klaus Ritzberger, Institute for Advanced Studies, Dept. of Economics, Stumpergasse 56, A-1060 Vienna, Austria 


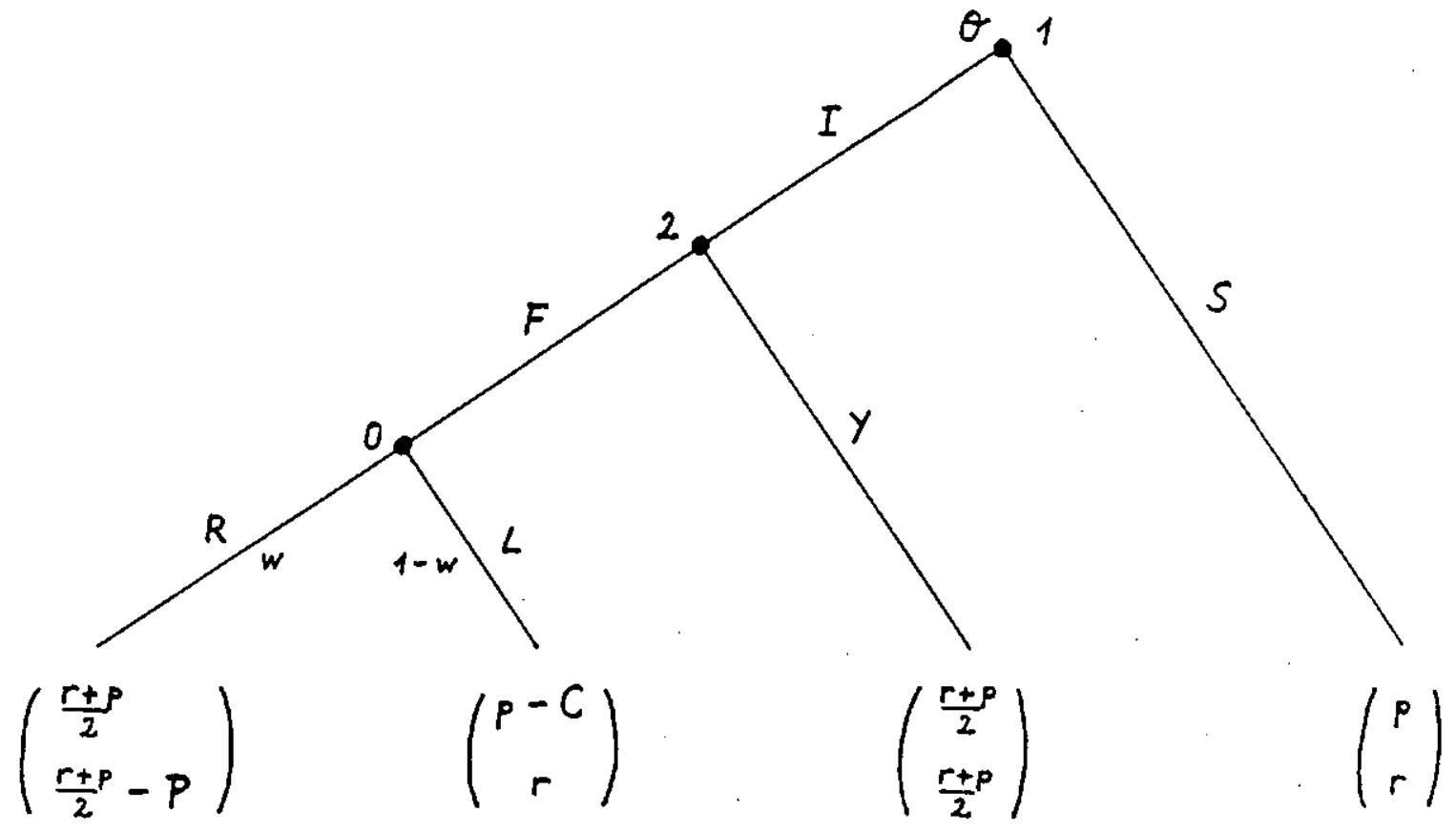

Figure II.1 


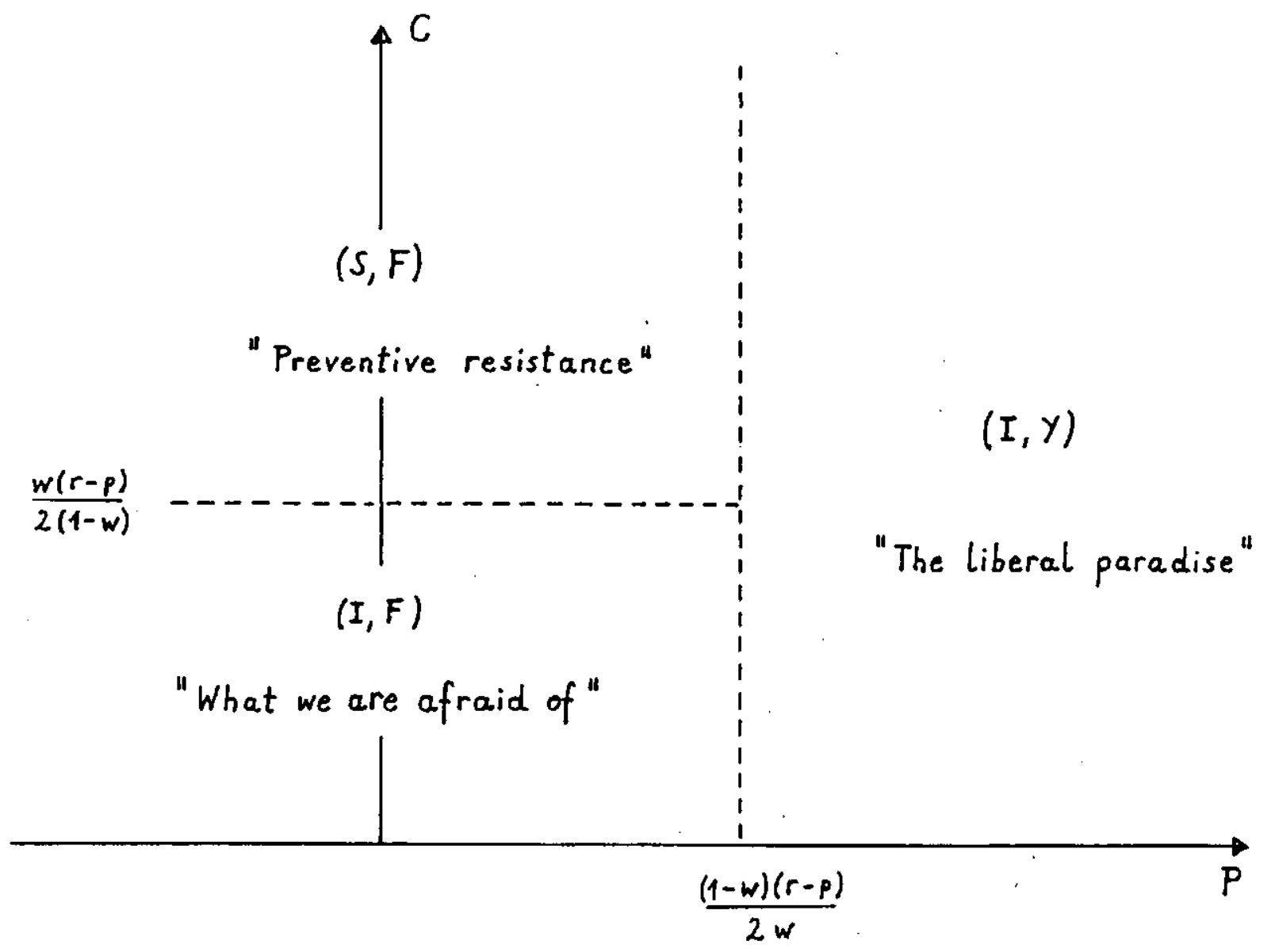

Figure II. 2 


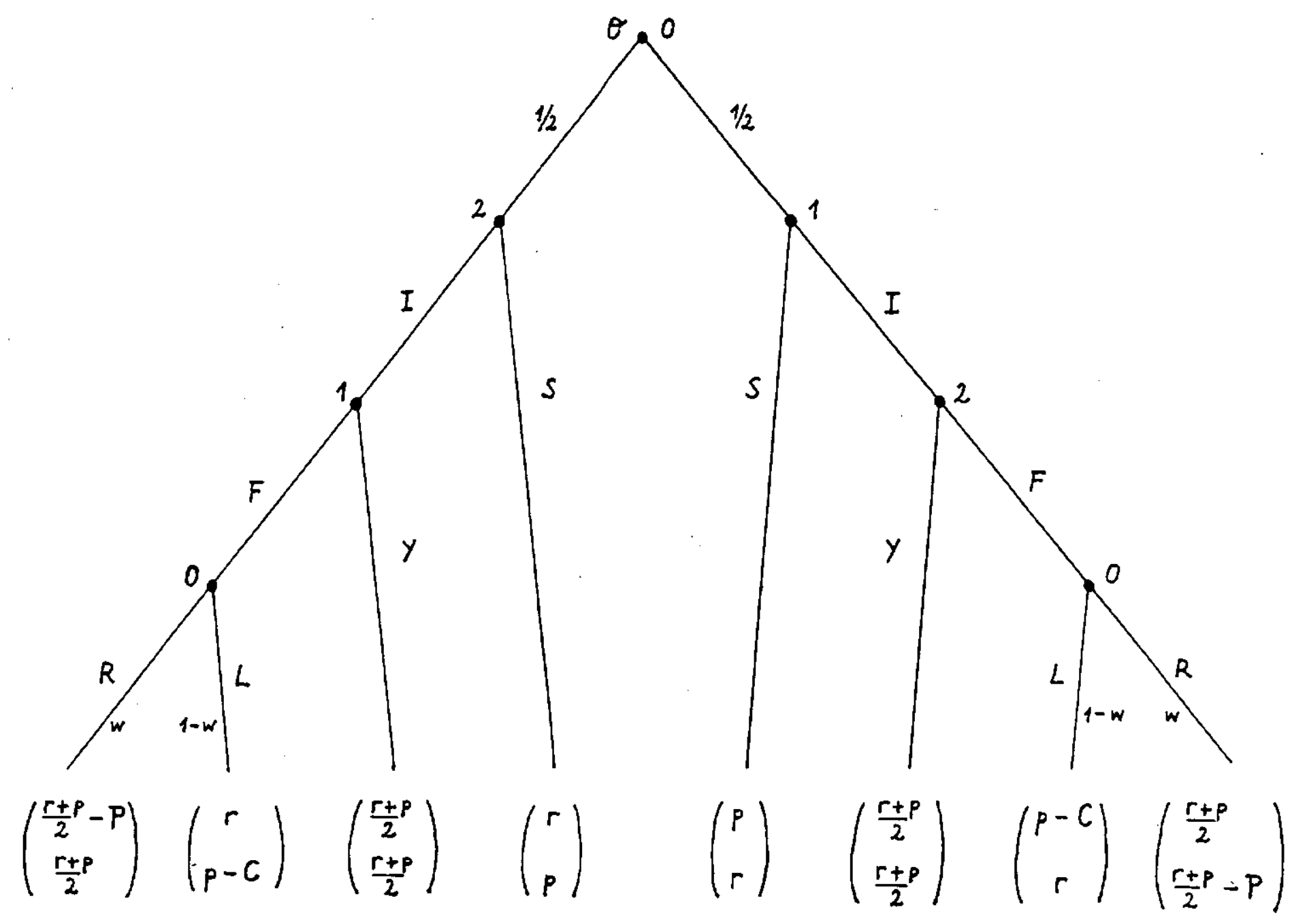

Figure III. 1 


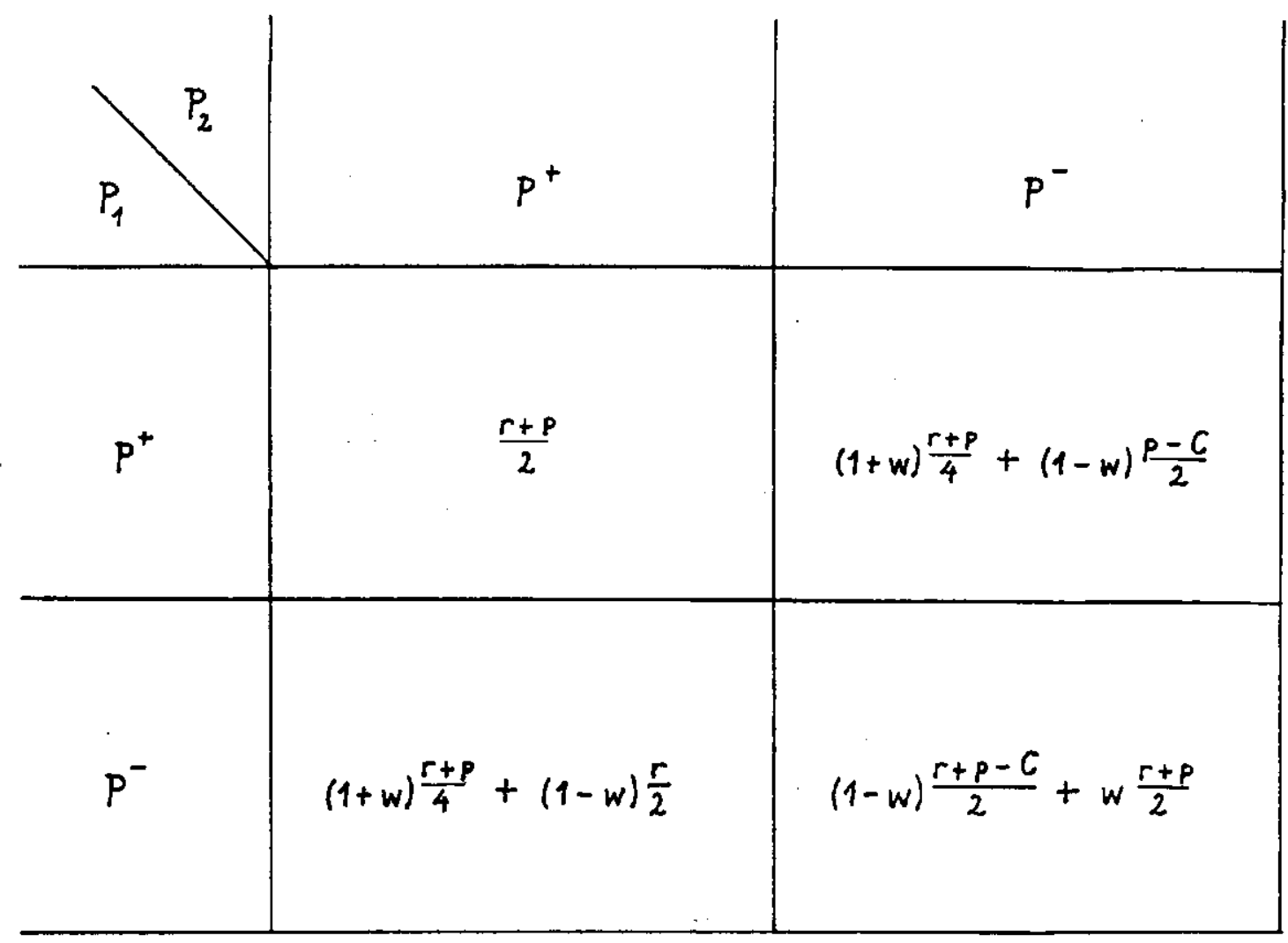

Table III.1 


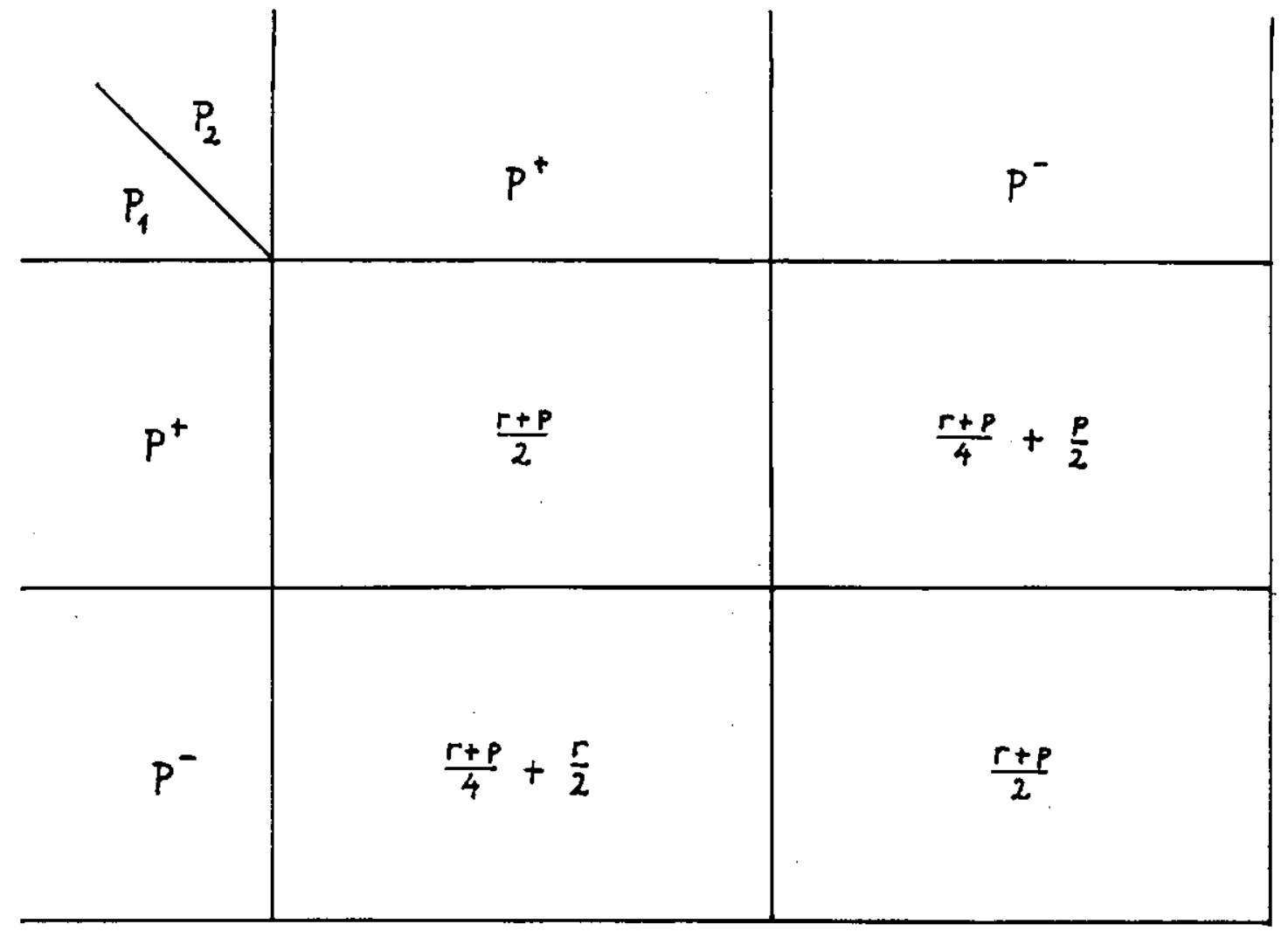

Table III.2 
- $111.8 .1-$

b) Genetically determined behavior

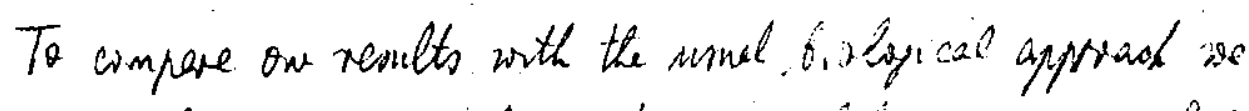

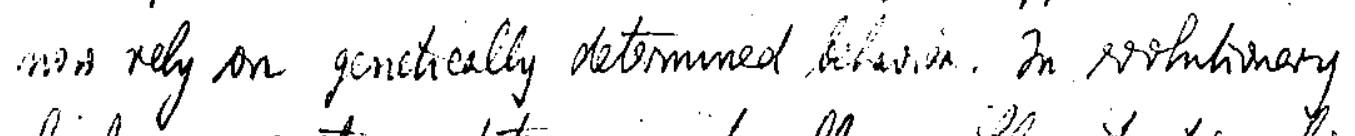

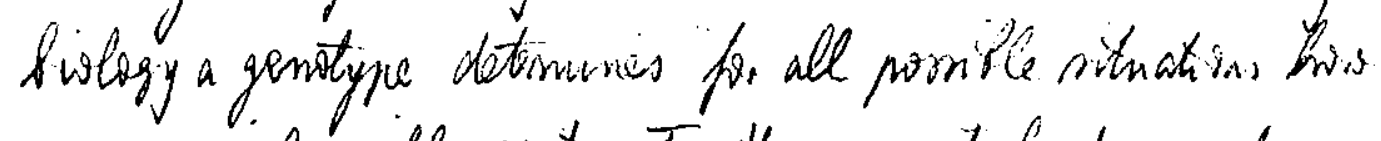
in annal will react. For the game it hand a serotine

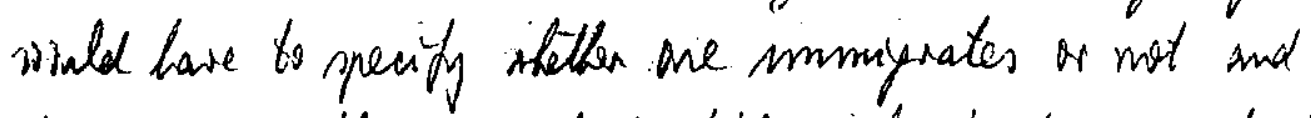

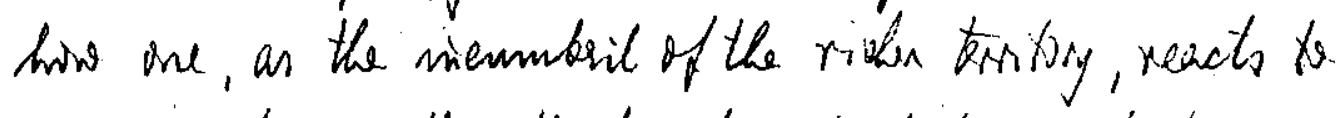

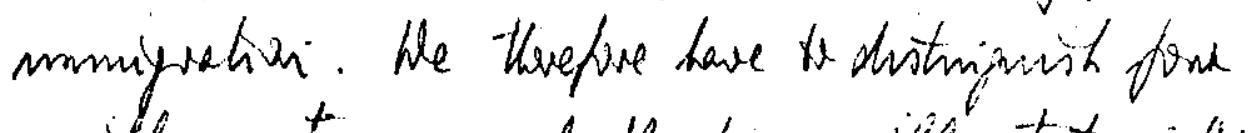

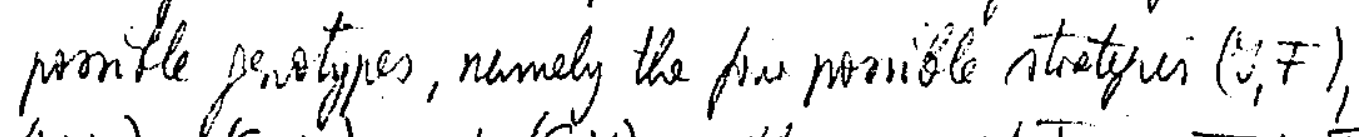
$(v, y),(S, F)$, and $(S, y)$ in the sane of Fy me IT.1. The set

$$
\delta=\{(T, F),(Y, y),(S, F),(S, y)\}
$$

is the strategy set or mutant set fir the Rolyiveal game when bicharion is gratrieally detoured.

As si the persons section me hes to derive the fires

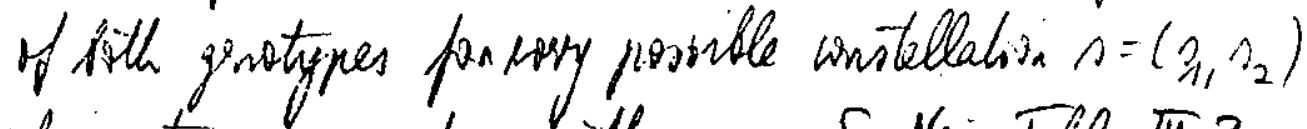
of grot types $s_{n}$ and $s_{2}$ with $s_{1} s_{2} \in S$. Nov Table III. 3 describes the normal form of the symmetric game in

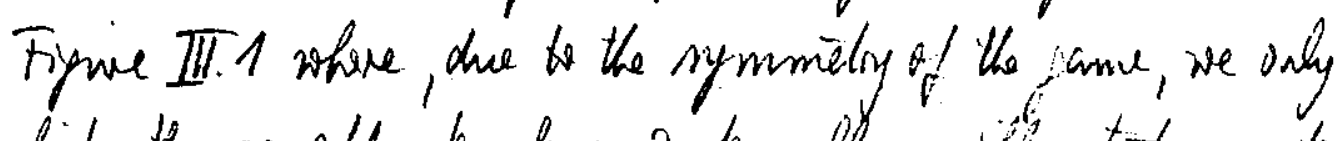

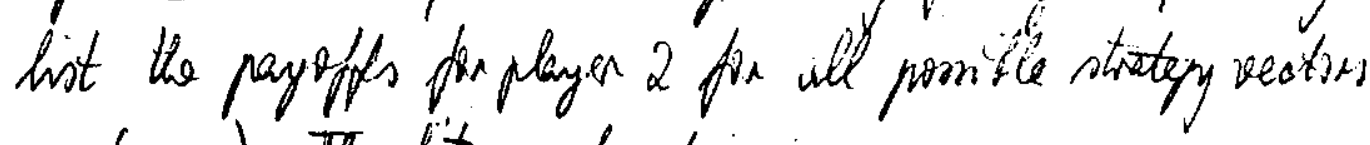
$s=\left(s_{1} s_{2}\right)$. The fit men function

$$
H: \begin{aligned}
S \times S \rightarrow \mathbb{R} \\
s=\left(s_{1}, s_{2}\right) \mapsto H(s)
\end{aligned}
$$

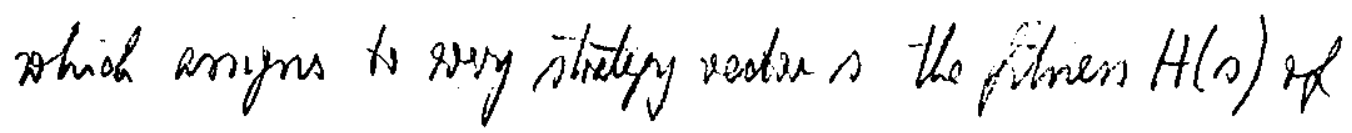




\section{Panitititions $(P=0)$ of ilonge 2}

\begin{tabular}{|c|c|c|c|c|}
\hline & $J, F$ & $4, y$ & $S, \bar{T}$ & \\
\hline$y_{1} F$ & $\begin{array}{l}\frac{1}{2}\left[w\left(\frac{x-r}{2}-P\right)+(1-w) r\right]+ \\
\frac{1}{2}\left[w \frac{x+n}{2}+(1-w)(\mu-C)\right]\end{array}$ & $\begin{array}{l}\frac{x+n}{4} \\
\frac{1}{2}\left[w \frac{x+\mu}{2}+(1-w)(p-C)\right]\end{array}$ & $\begin{array}{c}\frac{1}{2}\left[w\left(\frac{x+1}{2}-P\right)+(1-w) x\right]+ \\
\frac{1}{2}\end{array}$ & $+\frac{\frac{x+n}{4}}{2}$ \\
\hline $7, y$ & $\begin{array}{c}\frac{1}{5}\left[w\left(\frac{\gamma+n}{2}-p\right)+(1-w) x\right]+ \\
\frac{x+n}{4}\end{array}$ & $\begin{array}{l}\frac{\frac{x+n}{4}}{4} \\
+\cdots \frac{x+n}{4}\end{array}$ & $\frac{\frac{1}{2}\left[w\left(\frac{x}{2}-P\right)+(1-w) x\right]+}{\frac{n}{2}}$ & $\frac{\frac{\pi+n}{4}}{\frac{k}{2}}$ \\
\hline$S, F$ & $\begin{array}{c}\frac{\gamma}{2} \\
+\frac{1}{2}\left[w-\frac{\gamma+n}{2}+(1-w)(p-C)\right]\end{array}$ & $\frac{\frac{x}{2}}{2}\left[w \frac{x+n}{2}+(1-w)(\mu-C)\right]$ & $\begin{array}{r}\frac{x}{2} \\
+\frac{1}{2}\end{array}$ & $\begin{array}{r}\frac{x}{2} \\
+-\frac{n}{2}\end{array}$ \\
\hline$s_{1} y$ & $+\frac{\frac{x}{2}}{4}$ & $+\frac{\frac{r}{2}}{\frac{r+n}{4}}$ & $\begin{array}{l}\frac{x}{2} \\
+\frac{1}{2}\end{array}$ & $\begin{array}{r}\frac{x}{2} \\
+\quad \frac{p}{2}\end{array}$ \\
\hline
\end{tabular}

Fynse III.3 
-III. A.2-

plays 2 can be rumply dared firm Table III. 3 \&y setting $P=0$. Snick $P$ has no threat mijact on the rs and also no midiried mined if we rely on isetreally determined Behavior, one singly has forgetabint $P$ ancosdiny to the usual approach in sirhitionay sinology.

[inset Table III. 3 abinil hove]

Tow the frame of Table IIT.3 with $P=0$ mine can easily shine that $s=\left(s_{1}, p_{2}\right)$ with $s_{1}=(Y, F)$ dis $i=1,2$

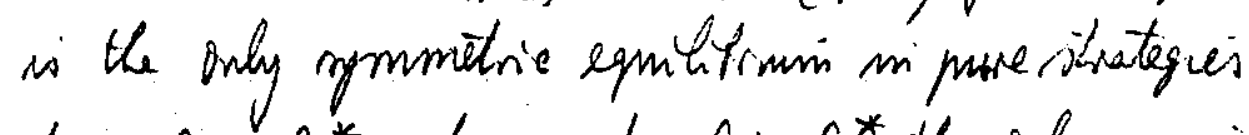
fir $C<C^{*}$ ootereas for $C>C^{*}$ the inlyngmmitis

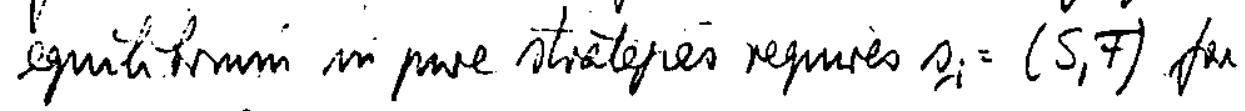
$i=1,2$. Since for $C<C^{*}$ the strategy $s_{2}=(0,7)$ is the orly best reply against $s_{i}=(y, F)$, the grovtyne is the inly sortintiviarily stat ie (a me) strategy for $C<C^{*}$.

Shive $\Omega_{2}=\left(S_{1} Y\right)$ is an altomatrie best reply. catania $s_{1}=(S, F)$ and mine $s_{2}=(S, F)$ and $s_{2}=(S, Y)$ we equally grid against $n_{n}=\left(S_{1} Y\right)$, the strategy toes not satisfy the second requirement (ii) of an solwtiverly stable strategy. To solve. the game of Table III. 3 with P. 0 also fir $C>C^{*}$, we can, houses, rely on the crassining of the concept

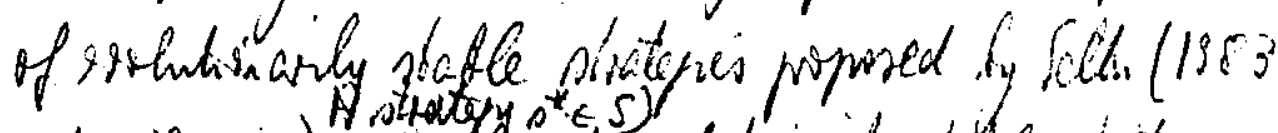

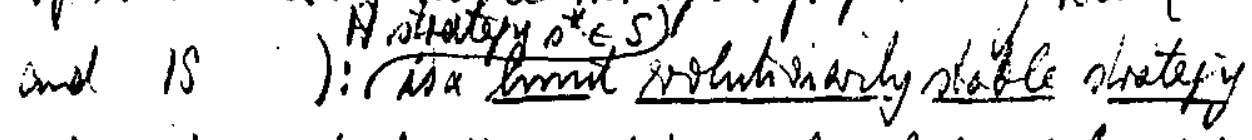

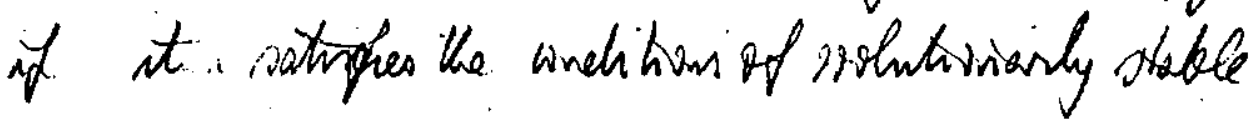


$-1116.9-$

shategrés for some shightly porturted parne. Tulike mi Selta (1975), hivesen, a prturbed doer not require that all possible mises have to be choses with poortive

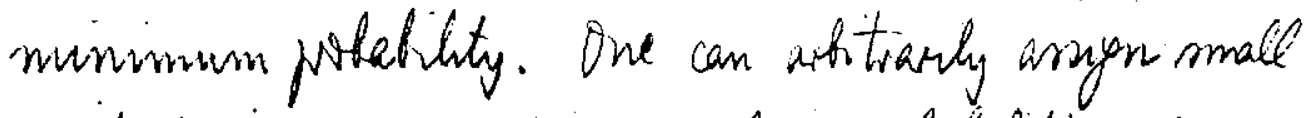
postwe or 2xo-numunum choice pobarilites. As a matter of frocl alss the mpirturited same is ine of its "shightly portwited games". Thin implies that epry srohtrinarily stable siratyy is also a timil. pothtivinarly statle strategy (Seltin, $198 \%$ ).

Arsume now por the game of Table IIT. with $P=0$ that enther $s_{n}=(y, 7)$ or $s_{1}=(y, y)$ lave to be wsed witt

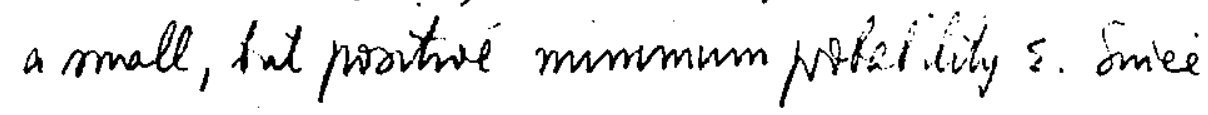

$$
\frac{1}{2}\left[w \frac{x+n}{2}+(1-w) x\right]>\frac{x+n}{4}
$$

is tive fin $x>p$ and $w<1$, the siriatery $s_{3}=(S, 7)$ is a better reply to the inised strategy og of playsit with $g_{1}(j, 7)$ on $g_{n}(v, y)=\Sigma$ and $g_{1}(s, 7)=1$ - $\varepsilon$ than $s_{z}=(S, Y)$. The to $C>C^{*}$ one can, furtlermire, sativify

$$
\frac{n}{2}>\varepsilon \frac{x+\mu}{4}+\frac{1-\varepsilon}{2}\left[w \frac{r+\mu}{2}+(1-w)(\mu-C)\right]
$$

by chiorniy $\varepsilon(20)$ small iningh. Whus sin the shightly protwed feme wotre $(y, \neq)$ or $(D, y)$ have to be uned at least with probarlity $\varepsilon$, it is a stict rymusistre equititinim to chose $(S, F)$ with masemial portakiting.

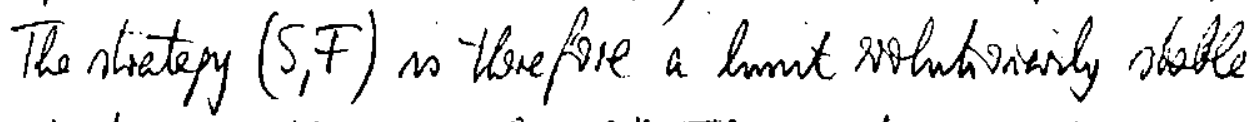
sitratefy in the renge $C>C^{*}$. Thus we have presed 
$-111.6 .4-$

Throrn 2: (i) The game of Table III. 3 with $P=0$ has a unquie sothtisiarily stadle (mue) stratesy, nemely $(y, F)$, if $C<C^{*}$.

(ii) Fir $C>C^{*}$ the same of Table III. 3 with P.O has iwo solutisinily stable (nuve) stretegy, but

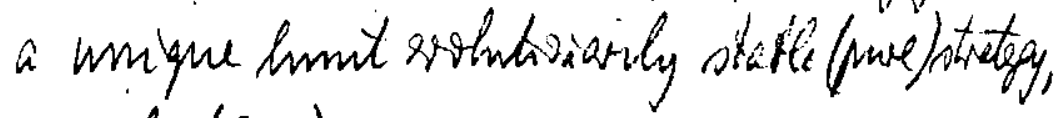
namely $(S, F)$.

Sure acousdny bo Therem 2 the solutiviessly stable rituation we etthen. "what we are afraid of " por $C<C$ " on "presentrie ressitance" for $C>C^{*}$, our cinchurion form Therem 1

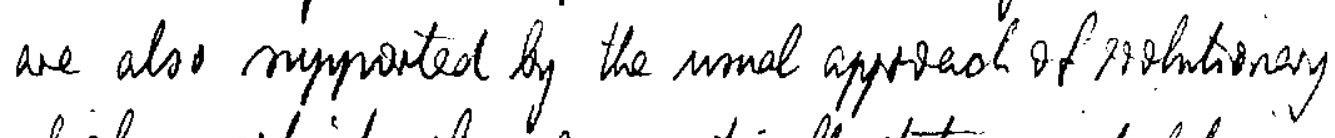
hology which rehes on gretically ctetermuned beharior. As

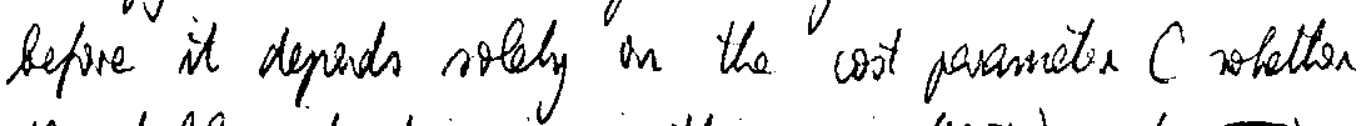
the stable situation is in the region $(y, F)$ ar $(S, T)$ of Fipwe II.2. The "hberal paradise" hivere, is mporritle repardien whether one aroumes genetieally determmed preferences on rehes on ginetreally determined beharios. 